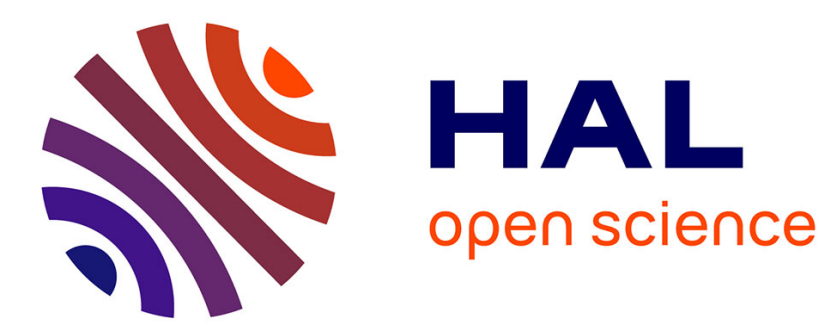

\title{
Bien-être et cadre de vie. Le regard des parents
} Lise Bourdeau-Lepage

\section{To cite this version:}

Lise Bourdeau-Lepage. Bien-être et cadre de vie. Le regard des parents. 2020. halshs-02431620

\section{HAL Id: halshs-02431620 \\ https://shs.hal.science/halshs-02431620}

Preprint submitted on 8 Jan 2020

HAL is a multi-disciplinary open access archive for the deposit and dissemination of scientific research documents, whether they are published or not. The documents may come from teaching and research institutions in France or abroad, or from public or private research centers.
L'archive ouverte pluridisciplinaire HAL, est destinée au dépôt et à la diffusion de documents scientifiques de niveau recherche, publiés ou non, émanant des établissements d'enseignement et de recherche français ou étrangers, des laboratoires publics ou privés. 
Pour citer le papier : Bourdeau-Lepage L. 2020, Bien-être et cadre de vie. Le regard des parents, document de travail HALSHS $n^{\circ} 02429791$. V2, 11 pages.

A paraître dans Chantal Zaouche Gaudron, Anne Dupuy et Christine Mennesson, 2020,"Bébé, petite Enfance en COntextes", ERES.

Rédaction finalisée V2 le 7 janvier 2020

\title{
Bien-être et cadre de vie. Le regard des parents
}

\author{
Bourdeau-Lepage Lise \\ Professeur de géographie \\ Université de Lyon, UMR 5600 EVS \\ $1 \mathrm{C}$ avenue des Frères Lumière \\ CS 7824269372 LYON CEDEX 08 \\ lblepage@gmail.com
}

\section{Résumé}

Ce papier se focalise sur le regard des parents en matière de cadre de vie et de niveau de bienêtre. Son objectif global est de révéler s'il existe des différences notables entre les habitants d'une métropole ayant des enfants à charge et ceux n'en ayant pas. D'abord, nous situons le contexte de notre étude en rappelant les transformations actuelles qui contribuent à modifier les aspirations et les besoins des citadins. Ensuite, nous présentons la méthodologie utilisée pour saisir les éléments potentiellement constitutifs du bien-être des individus. Par la suite, nous mettons en évidence les différences dans les éléments de bien-être révélées à partir des déclarations des individus en fonction de leur statut parental. Nous montrons que les parents sont plus sensibles à certaines aménités en milieu urbain que d'autres personnes. Nous analysons également les différences de préférences entre les personnes ayant des enfants à charge habitant, un quartier privilégié, le $6^{\mathrm{e}}$ arrondissement de Lyon et ceux habitant un quartier socialement hétérogène, le $7^{\mathrm{e}}$ Nord de Lyon. Cela nous permet de conclure sur le regard que porte les parents sur leur cadre de vie.

Mots-clefs : bien-être, cadre de vie, Lyon, parents, non parents.

\section{Introduction}

Le bien-être donne lieu à de nombreux travaux que l'on peut diviser en deux grandes catégories. Les premiers sont ceux qui s'intéressent au bien-être des personnes en général. Les différences entre ces travaux résident en grande partie, dans leur conception du bien-être : hédoniste, eudémonique, subjectif, objectif; leur manière d'appréhender le bien-être : universaliste, contextualisé ou capabiliste ; leur échelle d'analyse : nationale, régionale, urbaine (Maslow, 1943 ; Cantril, 1965 ; Andrews et al., 1976 ; Bailly, 1981 ; Sen, 1985 ; Kahneman et al., 2004 ; Cox et al., 2010 ; Bourdeau-Lepage et Tovar, 2011). La seconde catégorie regroupe ceux qui étudient le bien-être de populations spécifiques : les personnes âgées (Lefëbvre, 2010), les travailleurs (Cottraux, 2013), les jeunes adultes, les étudiants, les enfants (Gorza et Bolter, 2012 ; Junier, 2015), etc. Le bien-être des enfants est ainsi analysé dans le cadre éducatif (Boyer, 2010; Bougère, 2010; Bacro et al., 2017 ; Pinel-Jacquemin et Zaouche Gaudron, 2017 ; Fenouillet et al., 2017), au sein de la famille (Condran et Furstenberg, 1994 ; 
Delaunay-Guivardc'h, 2010 ; Gouttenoire, 2010 ; Barlow et al., 2015 ; Rees, 2017) ou entre pays (Smeeding et Rainwater, 1994 ; Andamson, 2010 ; Chzhen, 2016).

Force est de constater que peu de recherches se focalisent sur le regard des parents en matière de cadre de vie et d'éléments de bien-être. Pourtant, la parentalité, comme la jeunesse ou la vieillesse, peut être envisagée comme un élément spécifique influençant les aspirations et les besoins des personnes, transformant leur conception du bien-être et les éléments y contribuant. En effet, des études ont montré que les jeunes parents changent leur habitude de vie. Ils sortent moins et font moins de sport qu'avant la venue de leur enfant (Castelain-Meunier, 2013) ou déclarent une diminution de leur niveau de bien-être physique et mental (Dwenda et al., 2003). D'autres travaux montrent qu'au fil du temps, aux Etats-Unis, les parents sont plus heureux que les non parents mais surtout qu'ils sont plus socialisés et voient plus leurs amis. Ils soulignent également que leur famille agit comme un manteau protecteur envers le monde extérieur : la société, l'économique et la politique (Ifcher et Herbst, 2014).

Analyser les éléments considérés par les parents comme essentiels pour leur bien-être et voir s'il existe des différences notables en France entre les habitants d'une métropole ayant des enfants à charge et ceux n'en ayant plus ou pas, semble donc pertinent à plusieurs égards. Premièrement, cela contribue au débat sur le bien-être des parents. Deuxièmement, cela aide à comprendre comment se construit le bien-être sur un territoire urbain pour deux types de populations. Troisièmement, cela permet d'envisager des aménagements ou des mesures de politiques publiques ciblés envers les habitants ayant des enfants pour améliorer leur bien-être. Par conséquent, dans ce chapitre, nous nous positionnerons du côté des parents et révélerons les éléments constitutifs de leur bien-être dans deux arrondissements de Lyon aux caractéristiques socio-économiques différentes.

D'abord, nous situerons le contexte de notre étude en rappelant les transformations actuelles qui contribuent à modifier les aspirations et les besoins des citadins (Section 1). Ensuite, nous présenterons la méthodologie utilisée pour saisir les éléments potentiellement constitutifs du bien-être des individus (Section 2). Par la suite, nous mettrons en évidence les différences dans les éléments de bien-être révélées à partir des déclarations des individus en fonction de leur statut parental (section 3). Nous répondrons à la question suivante : les parents sont-ils plus sensibles à certaines aménités en milieu urbain que d'autres personnes (section 3.1) ? Nous analyserons aussi les différences de préférences entre les personnes ayant des enfants à charge habitant, un quartier privilégié, le $6^{\mathrm{e}}$ arrondissement de Lyon et ceux habitant un quartier socialement hétérogène, le $7^{\mathrm{e}}$ Nord de Lyon (Section 3.2). Cela nous permettra de conclure sur le regard que porte les parents sur leur cadre de vie.

\section{De l'importance du contexte sur la formation des aspirations et des désirs des citadins}

Les aspirations des personnes sont situées dans le temps (Chobart de Lauwe, 1964) et l'espace. Ce sont d'une certaine manière des construits sociaux. Par conséquent, l'étude que nous menons sur les préférences des individus en matière d'éléments de bien-être nécessite, au préalable, de définir le contexte dans lequel elle se déploie. Cette mise en perspective nous permettra de mieux comprendre, les désirs actuels des personnes et leurs attentes, en matière de cadre de vie.

\section{La globalisation de l'économie et ses effets}

Le premier élément de contexte a relevé est que nous vivons dans un monde globalisé. De nombreux changements ont lieu, transformant notre manière de créer, d'échanger, de consommer et d'habiter. D'abord, la révolution de l'information et de la communication, permise par la diffusion des nouvelles technologies de l'information et de la communication 
(NTIC) et l'émergence de la proximité virtuelle constituent un bouleversement technologique de premier ordre. Ensuite, les changements politiques et l'ouverture aux échanges de certains pays comme la Chine ou la Russie modifient la géopolitique mondiale. Enfin, l'économie se transforme à travers la baisse des coûts de transaction et la dérégulation des activités économiques, la dématérialisation mais aussi la différenciation des biens et des services. Ces phénomènes se combinent pour recomposer et globaliser l'espace économique. La production, la consommation et les échanges sont alors intégrés à une échelle planétaire de manière quasiinstantanée. Cette mutation a des effets sur notre manière d'habiter. En effet, elle génère un renforcement de la polarisation des activités et des populations (Huriot et Bourdeau-Lepage, 2009). Dans ce mouvement, la ville devient alors le cadre de vie de la plupart des Français. Or, les conditions urbaines peuvent provoquer chez l'individu des émotions négatives, le mettant en situation de mal-être. Confronté à un excès de stimuli (Moser, 2009), qui réduit sa capacité à traiter l'information (Milgram, 1970) et à échanger avec les autres, l'individu est peu enclin à l'altruisme (Simmel, 1903). Il peut alors avoir des difficultés à tisser des relations sociales en raison de cette surcharge environnementale. Cela va, dans certains cas, le conduire à rechercher le contact avec ses semblables en fréquentant des lieux de récréation comme les espaces verts. A cette surcharge environnementale liée aux conditions urbaines, peut s'ajouter une surcharge virtuelle.

\subsection{Accélération des rythmes et conscience écologique}

Cette surcharge virtuelle est liée aux effets de l'utilisation des NTIC, par les individus, dans tous les domaines de leur vie, sur leur manière de vivre-ensemble et sur leur lien avec la nature. Les NTIC permettent des interactions à très longue distance, étendant les réseaux sociaux. Elles permettent à l'individu à travers les réseaux virtuels d'être en relation continue avec d'autres. Cependant, cette compagnie l'oblige à être en représentation et à répondre aux multiples sollicitations. Cette instantanéité qui caractérise les NTIC entraîne une culture de l'immédiateté (Aubert, 2003). Les relations humaines et leurs formes se transforment. L'homme devient hypermoderne (Aubert, 2003). Dans ce mouvement, la sociabilité et l'intimité se reconfigurent. Le rapport au monde, au temps, à l'autre et à soi-même évolue.

La présence constante du virtuel dans toutes les sphères de la vie, la nécessité de s'adapter en continu, de répondre à l'urgence et l'accélération des rythmes génère un besoin de ralentir (Jauréguiberry, 2003). Le ralentissement passe par la déconnexion ou l'utilisation réfléchie des NTIC (Garcia et al., 2017), la reconquête de soi, la recherche de contacts en face à face (Jauréguiberry, 2014) et la reconnexion à l'environnement naturel. L'accélération des rythmes et la présence constante de la sphère virtuelle, peuvent aussi générer de l'entre soi. L'individu choisit alors les personnes avec qui, il souhaite interagir, et avec qui vivre.

Parallèlement à ce mouvement, on constate plusieurs prises de conscience de la part des individus : l'effet de l'activité humaine sur l'environnement et la finitude des ressources. Des accords internationaux pour préserver la biosphère sont signés ainsi que leurs traductions nationales et locales comme le Grenelle 2 ou la loi ALUR. La participation citoyenne, l'économie sociale et solidaire, l'économie circulaire se déploient. De nouveaux modes de vie, le fait maison, l'agriculture biologique, les circuits courts sont promus.

Dans ce contexte, les aspirations des personnes se modifient. L'homo oeconomicus semble se muer en homo qualitus : "Un homme qui cherche à maximiser son bien-être matériel et immatériel et fait de la satisfaction de son désir de nature et de la préservation de son environnement un des éléments constitutifs de son bien-être » (Bourdeau-Lepage, 2019). Les aspirations des personnes en matière de cadre de vie se métamorphosent. Un contact régulier avec la nature est recherché. Les activités de plein air, comme la marche, le jardinage sont plébiscités et les mouvements slow down émergent. 
Le contexte de notre étude présenté et en particulier, les transformations sociales en cours, il nous faut maintenant définir les éléments qui sont potentiellement constitutifs du bien-être des individus et décider de la méthode que nous souhaitons utiliser pour les révéler.

\section{Révéler les préférences des individus selon leur statut parental : éléments de méthode}

Nous admettons l'existence d'une variation des préférences des individus en matière d'éléments contribuant au bien-être. Fort de cette hypothèse, nous suivons la méthodologie développée par Bourdeau-Lepage et al. (2018) pour déterminer les éléments potentiellement constitutifs du bien-être sur un territoire. Cette méthodologie s'appuie sur une méthode d'analyse multicritère « alternative ». Elle se compose d'un panel d'éléments contribuant potentiellement au bien-être, qui mobilise les enseignements de l'économie urbaine, le rôle des aménités, la question de la liberté, du vécu et des opportunités sur le bien-être des individus. Ce panel est présenté aux enquêtés sous forme d'un jeu de cartes.

\subsection{Le jeu de carte}

Plusieurs raisons ont motivé ce choix. Le jeu de cartes permet une présentation simultanée de l'ensemble des critères, offrant ainsi une vision globale et concrète du panel d'éléments possibles. Son aspect ludique incite les personnes à répondre au questionnaire. Facile d'utilisation, il augmente le nombre de personnes capables de répondre au questionnaire. Il réduit le temps de passation du questionnaire et le risque de mettre les personnes dans une situation stressante. Notre jeu comprend 29 cartes, représentant chacune une aménité, historique, naturelle ou sociales notée $E B E_{k}$ avec $k=1$ à 29 (Cf. tableau 1).

Tableau 1 - Panel des éléments potentiellement constitutifs du bien-être sur un territoire $E B E_{k}$

\begin{tabular}{|l|l|}
\hline \multicolumn{1}{|c|}{ Aménités historiques } & \multicolumn{1}{c|}{ Aménités naturelles } \\
\hline Proximité au lieu de travail & Paysage naturel \\
Emplois type 1 & Environnement sain et sans nuisance \\
Emplois type 2 & Protection contre les risques naturels et technologiques \\
Stabilité de l'emploi sur le territoire & Protection et mise en valeur des espaces naturels \\
Salaires qu'offre le territoire & Accessibilité aux espaces naturels de loisirs \\
\cline { 2 - 2 } Qualité du logement & \multicolumn{1}{c|}{ Aménités sociales } \\
\hline Accessibilité à des équipements culturels et & Niveau de sécurité \\
sportifs & Diversité des professions des habitants \\
Accessibilité aux services d'aide à la personne et & Espaces communs où les gens se rencontrent et \\
aux familles & partagent des activités \\
Accessibilité aux services de santé & Associations et clubs où l'on peut s'investir et rencontrer \\
Accessibilité aux bars et restaurants & du monde \\
Accessibilité aux commerces et réparateurs & Manifestations locales, marchés, brocantes... \\
Accessibilité aux établissements scolaires & Prise de décision et participation au projet territorial \\
Couverture réseau internet et téléphone de bonne & Possibilité de rencontrer un compagnon ou une \\
qualité & compagne sur le territoire \\
Accessibilité du territoire & Aisance des habitants \\
Patrimoine typique & Niveau d'éducation \\
\hline
\end{tabular}

Source : Auteurs, adapté de Bourdeau-Lepage et al., 2018.

\subsection{Le protocole et la population enquêtée}

Le jeu de 29 cartes a été présenté, au printemps 2017, à un échantillon représentatif en âge et genre de la population des $6^{\mathrm{e}}$ et $7^{\mathrm{e}}$ arrondissements de Lyon ( $C f$. tableau 2). Dans un premier temps, la personne interrogée a été invitée à choisir, parmi les 29 cartes présentées sur un 
tableau devant elle, les dix les plus importantes pour que son niveau de bien-être soit le plus élevé possible. Dans un deuxième temps, il lui a été demandé d'ordonner les dix cartes retenues $E B E_{k i}$ avec $i$ : personne interrogée de 1 à 240, de la plus importante à la moins importante. Dans un troisième temps, il lui a été donné cent jetons représentant son niveau de bien-être, à répartir sur chacune des cartes retenues en respectant la hiérarchie qu'elle a établie préalablement.

Tableau 2 - Profils des 240 personnes enquêtées à Lyon (6 et $7^{\mathrm{e}}$ Nord arrondissements) au printemps 2017

\begin{tabular}{|c|c|c|}
\hline & \\
\hline & $6^{\mathrm{e}}$ arrondissement & $7^{\mathrm{e}}$ arrondissement Nord \\
\hline Caractéristiques & Nombre d'individus & Nombre d'individus \\
\hline \multicolumn{3}{|c|}{ Âge } \\
\hline $20-34$ ans & 42 & 67 \\
\hline $35-49$ ans & 27 & 17 \\
\hline $50-64$ ans & 23 & 16 \\
\hline 65 ans et + & 28 & 20 \\
\hline \multicolumn{3}{|c|}{ Genre } \\
\hline Homme & 52 & 54 \\
\hline Femme & 68 & 66 \\
\hline \multicolumn{3}{|c|}{ Niveau de diplôme } \\
\hline Pas de diplôme, Certificat d'études, BEPC & 10 & 13 \\
\hline CAP, BEP, Baccalauréat & 30 & 30 \\
\hline $\mathrm{Bac}+2$ et sup. & 80 & 77 \\
\hline \multicolumn{3}{|c|}{ Situation familiale } \\
\hline Célibataire, divorcé, veuf & 59 & 67 \\
\hline Marié, pacsé, en concubinage & 61 & 53 \\
\hline \multicolumn{3}{|c|}{ Enfants à charge } \\
\hline Oui & 43 & 31 \\
\hline Non & 77 & 89 \\
\hline \multicolumn{3}{|c|}{ Revenus du foyer } \\
\hline $0-1500 €$ & 38 & 55 \\
\hline $1500-3000 €$ & 36 & 36 \\
\hline+ de $3000 €$ & 46 & 29 \\
\hline \multicolumn{3}{|c|}{ Catégorie socio-professionnelle } \\
\hline Artisans, commerçants et chefs d'entreprise & 3 & 2 \\
\hline Ouvriers & 3 & 5 \\
\hline Employés & 11 & 12 \\
\hline Professions intermédiaires & 18 & 11 \\
\hline Cadres et professions intellectuelles & 23 & 14 \\
\hline Retraités & 36 & 24 \\
\hline $\begin{array}{l}\text { Autres personnes sans activité } \\
\text { professionnelle (étudiants, chômeurs) }\end{array}$ & 26 & 52 \\
\hline
\end{tabular}

Source : Auteur à partir de l'enquête Bourdeau-Lepage et Texier, 2017. 


\section{Des préférences très nettes en matière d'éléments de bien-être et des différences entre les parents et les non parents}

Les choix exprimés par les 240 Lyonnais interrogés en matière d'éléments de bien-être mettent en évidence des préférences très marquées. Neuf cartes regroupent à elles seules plus de la moitié des points attribués par les Lyonnais enquêtés ( $C f$. Figure 1). Ces derniers expriment une préférence nette pour les aménités naturelles. Trois éléments apparaissent particulièrement valorisés : un environnement sain et sans nuisance, l'accessibilité aux espaces naturels pour les loisirs et le paysage naturel. Ainsi, les Lyonnais accordent une grande importance à la présence d'éléments naturels dans leur cadre de vie et au contact potentiel avec la nature. Peut-être car victimes des surcharges environnementales et virtuelles, ils rejoignent les aspirations de l'homo qualitus.

A l'inverse, les aménités sociales sont relativement délaissées par les Lyonnais. Cependant, une exception forte est notée : le niveau de sécurité des biens et des personnes qui est l'élément qui reçoit le plus de points après un environnement sain et sans nuisance. Les éléments d'accessibilité aux aménités sociales - aux espaces publics et aux manifestations locales sont également relativement valorisés. Les aménités historiques, sont représentées de façon plus équilibrée. Toutefois, de très fortes disparités s'observent. Les aménités dont l'orientation est économique sont peu valorisées, comme les emplois, type 2 ou les salaires offerts par le territoire. A l'inverse, et à l'instar de ce qui est observé pour les aménités sociales, les Lyonnais 
ont exprimé leur préférence pour certains éléments relatifs à l'accessibilité : l'accessibilité du territoire, l'accessibilité aux services de santé et la proximité au lieu de travail.

Figure 1 - Les préférences des Lyonnais en matière d'éléments constitutifs du bien-être au printemps 2017

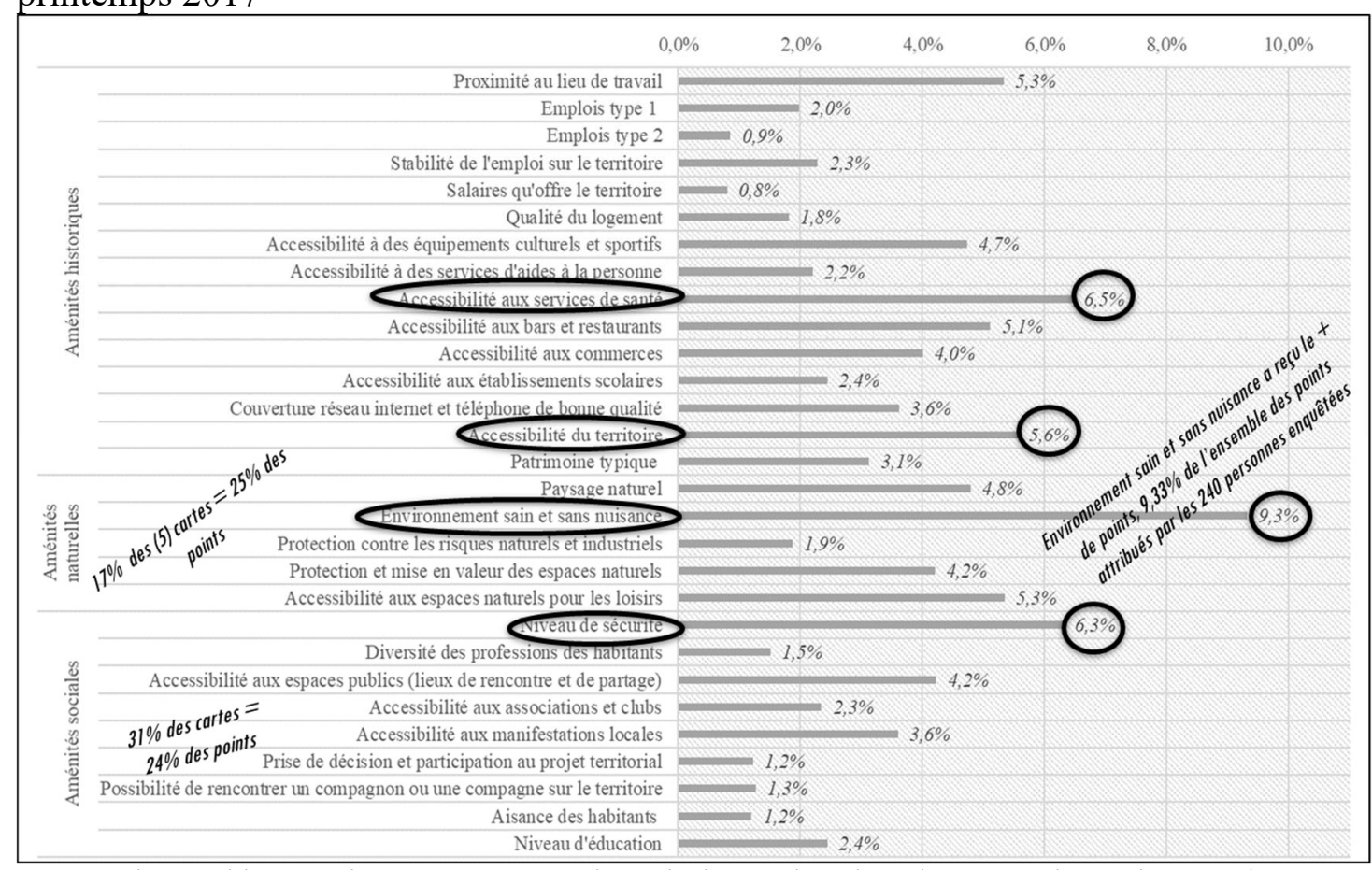

Lecture du graphique en barres groupées : Il s'agit du nombre de points accordés à chacune des 10 cartes ayant reçu le plus de points parmi les 29 cartes présentées aux 240 Lyonnais, exprimé en pourcentage du total des points. Ainsi, la carte « un environnement sain et sans nuisance » a reçu le plus de points, 9,3\% de l'ensemble des points attribués par les 240 personnes enquêtées.

\subsection{Les parents plus sensibles aux aménités historiques que l'ensemble des Lyonnais}

Quelques différences existent, de manière globale, en matière d'éléments préférés de bien-être entre l'ensemble des Lyonnais et les 74 Lyonnais ayant des enfants à charge. En effet, les Lyonnais avec enfants quel que soit leur quartier, ont une préférence nettement plus marquée pour l'accessibilité aux établissements scolaires que les Lyonnais en moyenne. Il en est de même pour l'environnement sain et sans nuisance, la sécurité des biens et des personnes et l'accessibilité aux services médicaux. De plus, ces Lyonnais considèrent la proximité du lieu de travail comme un élément de leur bien-être plus important que l'accessibilité du territoire (Cf. Figure 2). Globalement, ils sont plus sensibles aux aménités historiques que la moyenne des Lyonnais enquêtés en 2017. 
Figure 2 - Les préférences des Lyonnais avec enfants en matière d'éléments constitutifs du bien-être (10 éléments - mars-avril 2017)

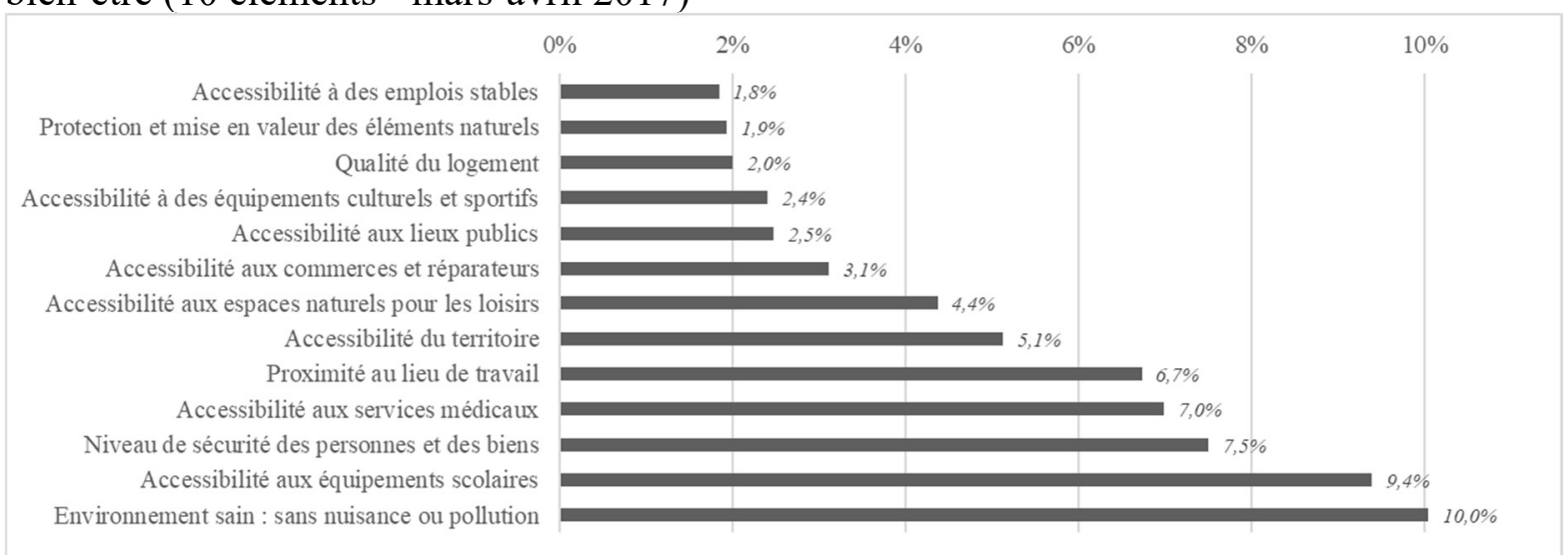

Lecture du graphique en barres groupées : Il s'agit du nombre de points accordés à chacune des 10 cartes ayant reçu le plus de points parmi les 29 cartes présentées aux 74 lyonnais avec enfants enquêtés, exprimé en pourcentage $\mathrm{du}$ total des points. Ainsi, la carte « un environnement sain et sans nuisance » a reçu le plus de points, $10 \%$ de l'ensemble des points attribués par les 74 personnes enquêtées.

Cependant, ces résultats cachent des différences entre les personnes ayant des enfants à charge. En effet, selon leur lieu d'habitation, le $6^{\mathrm{e}}$ arrondissement et le $7^{\mathrm{e}}$ Nord de Lyon, le rapport aux aménités est différent ( $C f$. figure 3 ).

\subsection{Le lieu d'habitation, un élément influençant les préférences des parents}

Les parents qui habitent le $6^{\mathrm{e}}$ arrondissement, quartier favorisé et bourgeois, mettent en premier élément de bien-être l'accessibilité aux équipements scolaires (11,4\%). Viennent ensuite un environnement sain et sans nuisance $(9,6 \%)$, le niveau de sécurité des biens et des personnes $(8,8 \%)$, la proximité au lieu de travail $(8,2 \%)$ et l'accessibilité aux services médicaux. Ils rejoignent ainsi, à une exception près, les préférences des Lyonnais.

Les parents du quartier du $7^{\mathrm{e}}$ Nord, quartier traditionnellement ouvrier, en cours de recomposition sociale, attirant une population jeune et diplômée, présentent des préférences plus variées et quelques peu différentes de ceux du $6^{\mathrm{e}}$. Le premier élément que ces parents retiennent est un environnement sain et sans nuisance (11,4\%). Viennent ensuite l'accessibilité aux établissements scolaires mais avec beaucoup moins d'importance que les parents du $6^{\mathrm{e}}$ puisqu'ils n'y consacrent que $6,8 \%$ des points totaux contre $11,4 \%$ pour ceux du $6^{\mathrm{e}}$. L'accessibilité aux services médicaux est presqu'aussi importante que l'accessibilité aux établissements scolaires pour les parents du $7^{\mathrm{e}}$ (elle récolte $6,7 \%$ du total des points). Les parents du $7^{\mathrm{e}}$ accordent moins d'importance au niveau de sécurité des biens et des personnes, que ceux du $6^{\mathrm{e}}$ arrondissement. 
Figure 3 - Les préférences des Lyonnais du $6^{\mathrm{e}}$ et du $7^{\mathrm{e}}$ arrondissement avec enfants en matière d'éléments constitutifs du bien-être (10 éléments - mars-avril 2017)

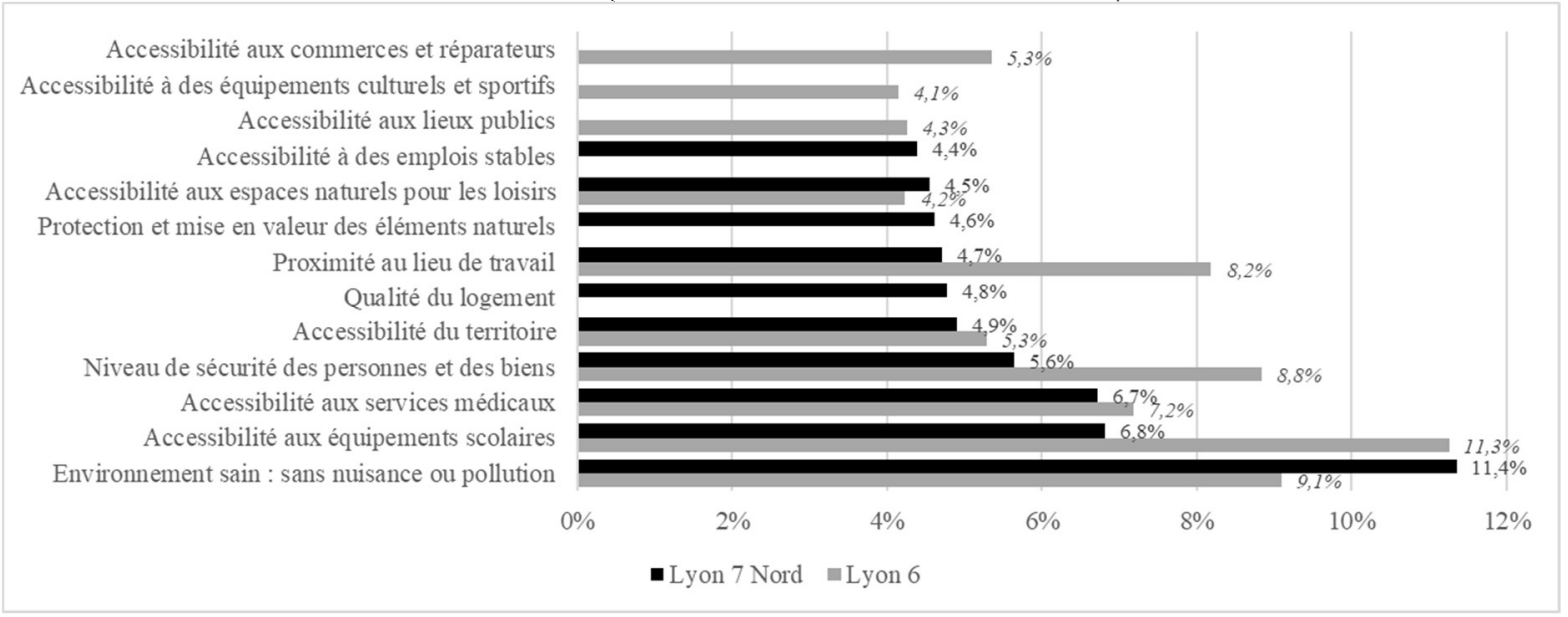

D'autres divergences sont observables. Ainsi, trois éléments apparaissent parmi les 10 plus importants des parents du $7^{\mathrm{e}}$ qui n'apparaissent pas au sommet de la hiérarchie des parents du $6^{\mathrm{e}}$. Il s'agit de la qualité du logement, de la protection et de la mise en valeur des éléments naturels et de l'accessibilité à des emplois stables.

Les parents qui habitent le $6^{\mathrm{e}}$ arrondissement considèrent que certains services sont importants pour leur bien-être alors que les parents $d u 7^{\mathrm{e}}$ ne les relèvent pas. Il s'agit en particulier des équipements culturels et sportifs, des commerces et réparateurs. Cette différence peut s'expliquer par la présence, dans ce ce quartier d'une population jeune, à dominante de cadres, au revenu particulièrement élevé et au taux de chômage assez faible. Cette population a a priori les moyens de consacrer une partie de son budget à ses loisirs. Elle est, semble-t-il, plus sensible à certains services et activités que peut lui offrir son territoire de vie. Peut-être avant tout parce qu'elle en a les moyens. Cela ne veut pas dire que les populations moins aisées du $7^{\mathrm{e}}$ arrondissement ignorent ces éléments mais plutôt qu'elles n'ont pas les moyens de les considérer. Les parents du $7^{\mathrm{e}}$ semblent adapter leur préférence en fonction de leurs possibilités. Ils mettent en priorité les éléments suivants : la qualité de l'environnement naturel de leur cadre de vie, l'accès à la santé et l'emploi. Les éléments liés à l'épanouissement personnel et aux activités récréatives ne sont pas avancés. Ils se focalisent sur la satisfaction de leur besoins fondamentaux. Nous retrouvons ici l'hypothèse de l'adaptation des préférences individuelles (Festinger, 1957 ; Elster, 1983) selon laquelle les individus se concentreraient sur ce à quoi ils peuvent prétendre, laissant de côté, les désirs qu'ils savent ne pas pouvoir assouvir.

\section{Conclusion}

A l'issue de cette analyse, nous pouvons répondre positivement à notre question initiale. Le statut parental a une influence sur les éléments qui sont importants dans la constitution du bienêtre des personnes. Les parents lyonnais sont plus sensibles que l'ensemble des 240 lyonnais enquêtés à certaines aménités comme l'accessibilité aux établissements scolaires et la qualité de leur environnement en termes de sécurité et d'éléments naturels.

Leur recherche en matière de cadre de vie devrait conduire les acteurs territoriaux a privilégié des aménagements qui permettent une présence et un contact fréquent avec les éléments naturels dans l'espace urbain. Les acteurs territoriaux devraient également être attentifs à assurer un bon maillage des établissements scolaires et des services de santé sur leur territoire. 


\section{Bibliographie}

ANDAMSON, P. 2010. Les enfants laissés pour compte : tableau de classement des inégalités de bien-être entre les enfants des pays riches, Unicef.

ANDREWS, F. CRANDALL, R. ANDREWS, RM. 1976. "The Validity of Measures of Self-Reported WellBeing", Social Indicators Research, 6/3-1, p. 1-19.

BACRO, F. GUIMARD, Ph. FLORIN, A. FERRIÈRE, S. GAUDONVILLE, T. 2017. « Bien-être perçu, performances scolaires et qualité de vie des enfants à l'école et au collège : étude longitudinale », Enfance, 1 (1), p. 61-80.

BAILLY, A., 1981. La géographie du bien-être. PUF (Espace et Liberté), Paris.

BARLOW J, BENNETT C, MIDGLEY N, LARKIN SK, WEI Y BARLOW J, BENNETT C, MIDGLEY N, LARKIN SK, WEI Y. 2015. "Parent-infant psychotherapy for improving parental and infant mental health", Cochrane Database of Systematic Reviews, Issue 1.

BOURDEAU-LEPAGE, L. 2019. « De l'intérêt pour la nature en ville. Cadre de vie, santé et aménagement urbain, Revue d'économie régionale et urbaine, 5, p. 893-911.

BOURDEAU-LEPAGE L, TOVAR E. 2011. "Well-being in the Paris region: widening regional disparities despite overall improvement", Metropolitics.

BOURDEAU-LEPAGE, L. et TEXIER P. 2017. Enquête sur le bien-être dans les espaces urbains denses L'exemple de Lyon, Projet BRRISE, Lyon, halshs-02428935, v1.

BOURDEAU-LEPAGE, L. TEXIER, P. CARRE, H. 2018. «Évaluer les déterminants du bien-être sur un territoire. Illustration à travers le cas d'une commune rhônalpine », Revue d'économie régionale et urbaine, 2018/4, 775-803.

BOYER, D., 2010. «Le maternage comme cadre de référence du bien-être de l'enfant », Informations sociales, 160(4), p. 6-9.

BROUGERE, G., 2010. «Le bien-être des enfants à l'école maternelle : Comparaison des pratiques pédagogiques en France et en Allemagne », Informations sociales, 160(4), p. 46-53.

CANTRIL, H. 1965. The pattern of human concerns. New Brunswick, Rutgers University Press.

CASTELAIN MEUNIER, Ch. 2013. Le ménage, la fée, la sorcière et l'homme nouveau, Paris, Stock.

CHOMBART DE LAUWE P.-H. 1964. "Aspirations, images guides et transformations sociales », Revue française de sociologie, 5-2. p. 180-192.

CHZHEN, Y. 2016. Mapping inequality for child well-being in rich countries, Unicef Connect, Data and Research.

CONDRAN, G. et Furstenberg, F. 1994. «Évolution du bien-être des enfants et transformations de la famille américaine », Population, vol. 49(6), p. 1613-1637.

COTTRAUX J. (éd.) 2013. Psychologie positive et bien-être au travail, Paris, Elsevier Masson.

COX D, FRERE M, WEST S, WISEMAN J. 2010. "Developing and using local community wellbeing indicators: Learning from the experience of Community Indicators Victoria”, Australian Journal of Social Issues, 4/201045/1, p. 71-88.

DELAUNAY-GUIVARC'H, V. 2010. «Focus - Contribuer au bien-être des enfants en soutenant leurs parents : Les actions des caisses d'Allocations familiales », Informations sociales, 160(4), p. 42-44.

DIENER, E. 1984. "Subjective Well-being”, Psychological Bulletin, 95, p. 542-575.

DODGE, R., Daly, A., Huyton, J. et Sanders, L. 2012. "The challenge of defining wellbeing”, International Journal of Wellbeing, 2 (3), p. 222-235.

DWENDA K. GJERDINGEN, BRUCE A. CENTER. 2003. "First-Time Parents' Prenatal to Postpartum Changes in Health, and the Relation of Postpartum Health to Work and Partner Characteristics", The Journal of the American Board of Family Practice July 2003, 16 (4) p. 304-311.

ELSTER, J. 1983. Sour Grapes: Studies in the Subversion of Rationality, Cambridge, Cambridge University Press.

FENOUILLET, F., CHAINON, D., YENNEK, N., LEMASSON, J. et HEUTTE, J. 2017. « Relation entre l'intérêt et le bien-être au collège et au lycée ». Enfance, 1(1), p. 81-103.

FESTINGER, L. 1957. A theory of cognitive dissonance, Stanford, Stanford university Press.

GORZA, M. et BOLTER, F. 2012. «Indicateurs de bien-être de l'enfant, une déclinaison en protection de l'enfance est-elle possible ? », Journal du droit des jeunes, 312(2), p. 26-36.

GOUTTENOIRE, A. 2010. «Focus - Le bien-être de l'enfant dans la Convention internationale des droits de l'enfant » Informations sociales, 160(4), p. 30-33.

HERBST, C. M., et IFCHER, J. 2016. "The increasing happiness of US parents", Review of Economics of the Household, 14(3), p. 529-551.

HURIOT, J.-M et BOURDEAU-LEPAGE, L. 2009. Economei des villes contemporaines, Paris, Economica.

JUNIER, H. 2015. « Alors heureux ? Mesurer le bien-être de l'enfant » dans V. Bedin (éd.), L'enfant et le monde : Psychologie de l'enfant. État des lieux, Auxerre, Editions Sciences Humaines, p. 117-119.

KAHNEMAN, D. KRUEGER, A. SCHKADE, D. SCHWARZ, N. STONE, A. 2004. "Toward National WellBeing Accounts", American Economic Review, 94/2, p. 429-434. 
LEFEBVRE, M. 2010. «Mesurer et comparer le bien-être des personnes âgées dans l'UE », Revue française d'économie, volume 25(1), p. 213-227.

MASLOW, A-H. 1943. “A theory of human motivation”, Psychological Review, 7/1943-50/4, p. 370-396.

MILGRAM, S. 1970. “The Experience of Living in Cities », Science,167(3924), p. 1461-1468.

MOSER, G. 2009. Psychologie environnementale. Les relations hommes-environnement, Bruxelles, de Boeck.

PINEL-JACQUEMIN, S. et ZAOUCHE GAUDRON, C. 2017. « Spécificités du bien-être scolaire des enfants en situation de précarité », Enfance, 1(1), p. 105-122.

REES, G. 2017. "Family structure and children's subjective well-being: A comparative analysis in eight European countries", Enfance, 1(1), p. 13-35.

RIFF, C. 1989. "Happiness is Everything, or is it? Explorations on the Meaning of Psychological Well-being", Journal of Personality and Social Psychology, 57, p. 1069-1081.

ROBERT, N. et FAVARO, M., 2013. «Les conflits éthiques en question », Note Scientifique de l'INRS, 312.

SEN, A. 1985. "Well-being, agency and freedom - the Dewey lectures 1984", Journalof Philosophy, 82, p. 169221.

SIMMEL, G. 1903. Métropoles et mentalités dans Y. GRAFMEYER et JOSEPH I (ed.) 1979, L'école de Chicago. Naissance de l'écologie urbaine, Paris, Editions du champ urbain, p. 61-77.

SMEEDING, T. et RAINWATER, L. 1994, « Le bien-être économique des enfants européens. Une perspective comparative », Population, vol. 49(6), p. 1437-1449.

STEILER, D. 2013. «La mindfulness en entreprise : bien-être et performance » dans J. COTTRAUX (éd.). Psychologie positive et bien-être au travail, Paris, Elsevier Masson, p. 131-152.

\section{Remerciements}

Cet article est issu des enquêtes menées dans le cadre du projet BRRISE. Le projet BRRISE est un projet de recherche du programme Pour et Sur le Développement Régional (PSDR4 Rhône-Alpes) qui a bénéficié d'un financement de l'INRA, de la Région Auvergne-Rhône-Alpes, de l'Irstea et de l'Union européenne via le FEADER dans le cadre du Partenariat Européen pour l'Innovation (PEI-AGRI). 